\title{
A chacun son risque
}

Par un postulat, la conseillère nationale d'alors avait demandé à ses actuels collègues du Conseil fédéral d'empêcher la création de «caisses bon marché». Son postulat fut refusé au motif des directives existantes de l'OFAS. Les doutes concernant la décision de la Mobilière Assurance de ne plus conclure d'assurance-voiture avec des intéressés venant des Balkans ou de l'Europe de l'Est ont également été vite dissipés à l'heure des questions au Conseil fédéral. En effet, il est juridiquement autorisé d'exclure ou de pénaliser financièrement des groupes d'assurés déterminés, pour autant que la procédure se fonde sur des statistiques fiables. Certains points de droit constitutionnel sont encore vérifiés. Deux tendances se font face: la compensation des risques garantie par l'Etat contre la sélection des risques toujours plus affinée sur le plan technique.

\section{Compenser, limiter...}

La loi sur la surveillance des assurances a été introduite en Suisse en 1885 pour protéger en premier lieu les assurés. Dans un pays où les habitants sont les mieux assurés, il est plus simple d'éviter les mauvais risques que d'augmenter les primes. Cela vaut principalement pour la branche en plein essor des assurances-vie créatrices de capitaux pour la prévoyance privée. De fait, la loi fédérale sur l'analyse génétique humaine règlera le droit du questionnement des assureurs privés à partir de 2007. Les possibilités d'examiner les risques seront limitées pour les assurances-vie et invalidité. Seront exclus les examens présymptomatiques, prénataux et génétiques pour la planification familiale. Les examens faits jusque là dans les cas non réglés par des exceptions et des interdictions de recherche devront être communiqués, pour autant qu'ils soient techniquement fiables et importants pour le calcul scientifique des primes. La LAMal introduite en 1996 avait aussi pour objectif de renforcer la solidarité entre assurés malades et bien portants ainsi qu'entre riches et pauvres dans le cadre de l'obligation de s'assurer avec le librepassage complet des assurés. Mais depuis, la LAMal est un chantier permanent et les programmes d'économie encouragent la politique de ségrégation par l'augmentation, à chaque révision, de la pression économique sur les assureurs. Désireux d'améliorer la situation, le Conseil des
Etats prévoit, par une meilleure compensation, de réduire l'envie de sélectionner les risques et de déplacer la concurrence sur les coûts.

\section{...ou sélectionner}

Les mathématiques actuarielles ont commencé par dresser des tables de mortalité. Aujourd'hui, les nouvelles technologies permettent une sélection des risques beaucoup plus précise, car le flux grandissant de données en provenance de tous les domaines de la vie permet théoriquement un contrôle quasi sans failles. La numérisation et les nanotechnologies ont fait irruption dans le quotidien: textiles et objets d'usage courant, processeurs et senseurs sur les étalages des magasins. La technique moléculaire permet une médecine prédictive d'une grande précision, capable d'énoncer les risques et de déclencher des mesures de surveillance à vie. En corollaire, on accepte l'effet secondaire paradoxal consistant à ce que chaque gestion de risques en entraîne de nouveaux. Ce développement incroyable procure peu d'avantages et beaucoup de risques nouveaux. Il suffit de penser à la mise en réseau de domaines les plus divers échappant à tout contrôle, comme les données biométriques, les empreintes digitales génétiques, la recherche d'indices par ordinateur, les contrôles vidéo, le profil du consommateur, les banques de données médicales et les payements en ligne. La rapidité des innovations dépasse les législateurs et leurs experts ainsi que les organismes responsables de la protection des consommateurs et des données. Celui qui tablera exclusivement sur le libre marché ne réagira guère contre la sélection des risques. En outre, il sera plus difficile de justifier des prestations communautaires dans un climat égoïste. Pourquoi les bons risques doivent-ils payer pour les mauvais risques? Que se passerat-il avec les personnes génétiquement défavorisées et sujettes à risques et avec celles qui refuseront les mesures de prévention officielles? $\mathrm{Ne}$ profitera-on d'un traitement que si l'on se conforme aux règles de la future méritocratique sanitaire? La hiérarchie des risques va-t-elle créer une nouvelle société féodale, ou plus simplement dit, que devra-t-on assumer pour le risque que l'on présente?

Erhard Taverna 